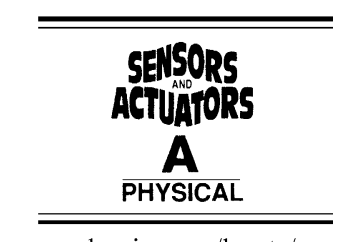

www.elsevier.com/locate/sna

\title{
A MEMS micromirror fabricated using CMOS post-process
}

\author{
Ying-Chou Cheng ${ }^{\mathrm{a}, *}$, Ching-Liang Dai ${ }^{\mathrm{b}}$, Chi-Yuan Lee ${ }^{\mathrm{c}}$, Ping-Hei Chen ${ }^{\mathrm{a}}$, Pei-Zen Chang ${ }^{\mathrm{d}}$ \\ a Department of Mechanical Engineering, National Taiwan University, Taipei, 106 Taiwan, ROC \\ b Department of Mechanical Engineering, National Chung Hsing University, Taichung, 402 Taiwan, ROC \\ ${ }^{\mathrm{c}}$ Department of Mechanical Engineering, Yuan Ze University, 320 Taoyuan, Taiwan, ROC \\ ${ }^{\mathrm{d}}$ Institute of Applied Mechanics, National Taiwan University, Taipei, 106 Taiwan, ROC
}

Received 8 June 2004; received in revised form 27 December 2004; accepted 7 February 2005

Available online 17 March 2005

\begin{abstract}
This work describes the fabrication of a micromachined micromirror by the conventional $0.35 \mu \mathrm{m}$ CMOS process and a simple maskless post-CMOS process. The micromirror contains a rectangular mirror plate and four pairs of serpentine supported beams, is integrated with a $1 \times 4$ demultiplexer and a four-stage charge pump circuits on a chip. Maskless dry and wet etching processes are the only requirement to suspend the structure. The primary limitation in the fabrication of microstructures has been overcome by the development of a hybrid processing technique, which combines both an anisotropic dry etch and an isotropic wet etch step. A highly reliable wet etching step with high selectivity between aluminum and sacrificial oxide is also reported. Experimental results reveal that the micromirror has a tilting angle of around $5^{\circ}$ at operation voltage of $22.5 \mathrm{~V}$ and a dynamic response less than $5 \mathrm{~ms}$. The surface properties of the CMOS micromirror, detailed process flows, measurement set-up and the experimental results are also presented in this work.
\end{abstract}

(C) 2005 Elsevier B.V. All rights reserved.

Keywords: MEMS; CMOS process; CMOS-MEMS

\section{Introduction}

The conventional CMOS process enables the cointegration on a single chip of mature integrated circuits with some smart mechanical microstructures. Various fabricating technologies, including LIGA, surface micromachining and bulk micromachining, have been proposed to meet particular industrial requirements. However, the specialized processes may not support the on-chip integration of MEMS devices and circuit. Therefore, the developments of a MEMS structure by a commercial CMOS batch fabrication process have attracted much attention $[1,2]$.

The advantages of using a micromachined micromirror are its low sensitivity to polarization and its functioning in a broad band. The development of MEMS-based mirrors for

\footnotetext{
* Corresponding author. Present address: Institute of Applied Mechanics (Lab. 433), National Taiwan University, 1, Sec. 4, Roosevelt Rd., Taipei 10617, Taiwan, ROC. Tel.: +886 2 33665646; fax: +886 226608560 .

E-mail address: yccheng@mems.iam.ntu.edu.tw (Y.-C. Cheng).
}

optical applications, including in projection display systems [3,4], optical scanners [5,6], variable optical attenuators [7-9] and other specific applications has attracted much interest recently [10]. Prior work on micromirror optical switching has demonstrated the feasibility of making surface or bulk micromachined micromirrors for light beams steering or for use in optical interconnect applications, by silicon deep etching or by the use of expensive SOI (silicon-on-insulator) wafers [11-14]. However, issues raised in the literature include high process complexity, high operation voltage or power consumption and process incompatibility with currently used CMOS process.

This work demonstrates a fully CMOS process compatible micromirror, which contains a mirror plate, a $1 \times 4$ demultiplexer and a four-stage charge pump circuits. The standard $0.35 \mu \mathrm{m}$ CMOS process and a completely CMOS compatible post-process procedure are utilized to fabricate the device. The proposed micromirror is electrostatically actuated and is driven by a square-wave with a $20 \mathrm{~Hz}$ repetition frequency. Experimental results indicate that the micromirror 
have a tilting angle of $5^{\circ}$ at a driving voltage of about $22.5 \mathrm{~V}$ according to the triangular relationship, and a dynamic response less than $5 \mathrm{~ms}$, as measured by a commercialized LDV system. The surface properties of the micromirror, detailed process flows, measurement set-up and experimental results are also presented.

\section{Micromirror structure design}

The device proposed herein is a micromachined micromirror using the conventional CMOS process. A $10 \times 10 \mathrm{mi}-$ cromirror array with on-chip circuits, as shown in Fig. 1, is designed as a testboard for examining the feasibility study of a fully CMOS process compatible micromirror. Therefore, this work investigates the integrating compatibility between standard CMOS process and MEMS devices, the surface quality of mirror plate after post-processing, and the measurement of the dynamic response of a micromirror. However, the total system mentioned previously, a $10 \times 10$ mirror array, is currently under test and development. Possible applications of the MEMS-based micromirrors include the optical attenuators, precise position sensors, high pixel density display without add-on bonding process or short range data transmission.

Electrostatic actuation is used to deflect the micromirror. Fig. 2(a) depicts the structure and dimensions of a mirror. It contains a top mirror plate with an aluminum layer with a thickness of $1 \mu \mathrm{m}$, four bottom-fixed electrode plates and four pairs of supported beams. The top mirror plate is a movable electrode plate, and four bottom-fixed electrode plates are located under each side of the mirror plate. The gap between the fixed-electrode plate and mirror plate is about $5.6 \mu \mathrm{m}$. The mirror includes four sets of parallel-plate electrodes, formed from four fixed-electrode plates and the top mirror plate. The supported beams are S-shaped serpentine structures, as indicated in Fig. 2(b). One end of the supported beams is fixed to an anchor, which is stacked using metal layers and tungsten

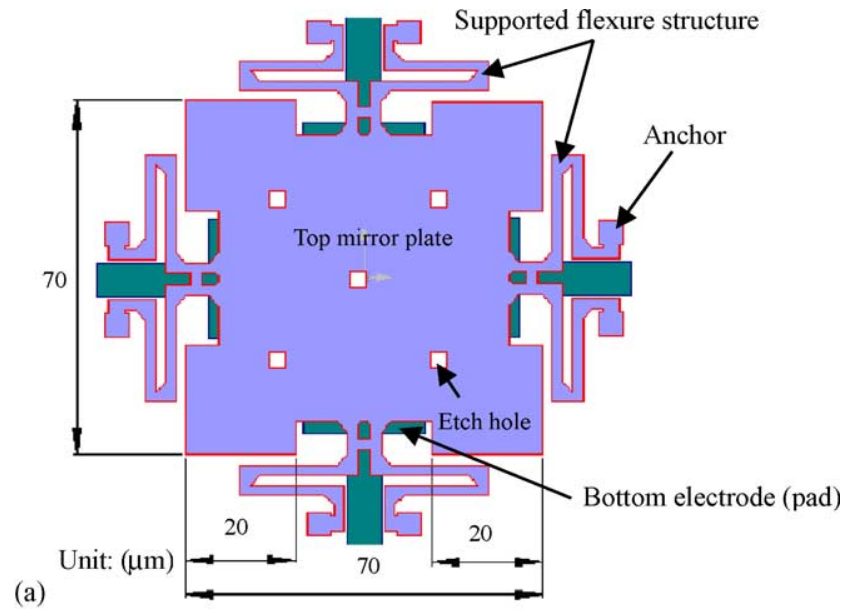

(b)

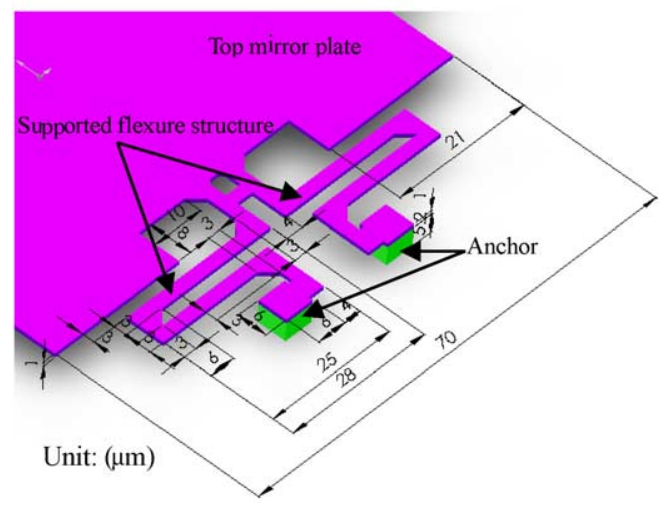

Fig. 2. Structure and dimensions of a micromirror structure: (a) mirror plate and fixed bottom electrodes and (b) flexural supported beams.

plugs (vias), and the other end is attached to the top mirror plate. The mirror plate is about $70 \mu \mathrm{m} \times 70 \mu \mathrm{m}$ areas and is about $1 \mu \mathrm{m}$ thick. The area of each fixed-electrode plate is $25 \mu \mathrm{m} \times 28 \mu \mathrm{m}$. The mirror plate and the supported beams used in the CMOS process are all made from metals. All anchors comprise stacked metal and via layers. The top mirror plate is electrically connected to highly phosphorous-doped

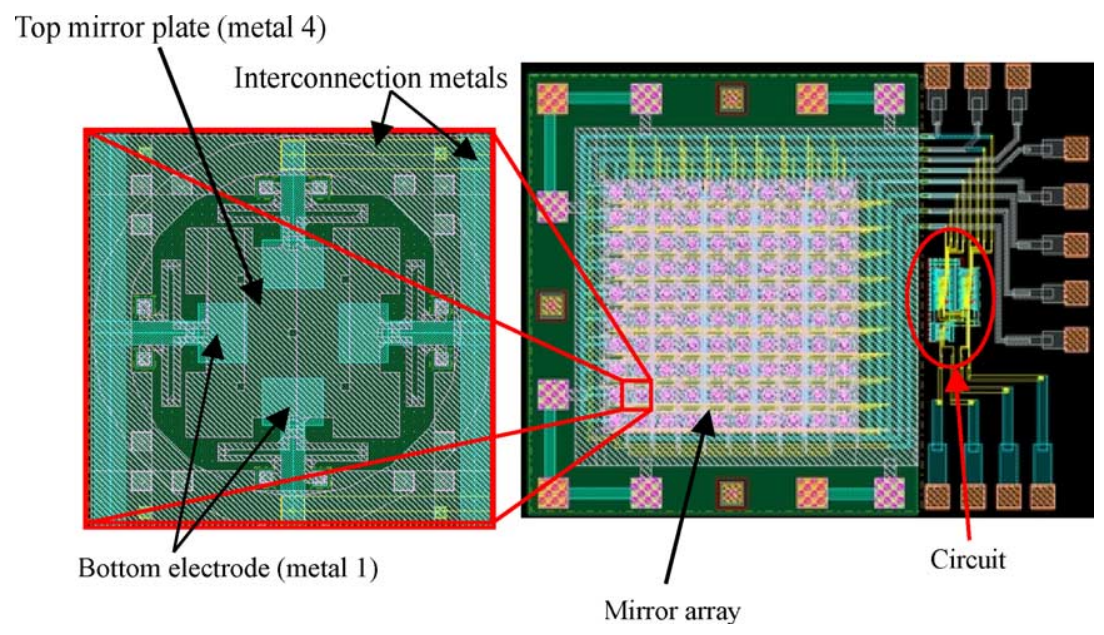

Fig. 1. Layout of the micromirror array with circuits. 


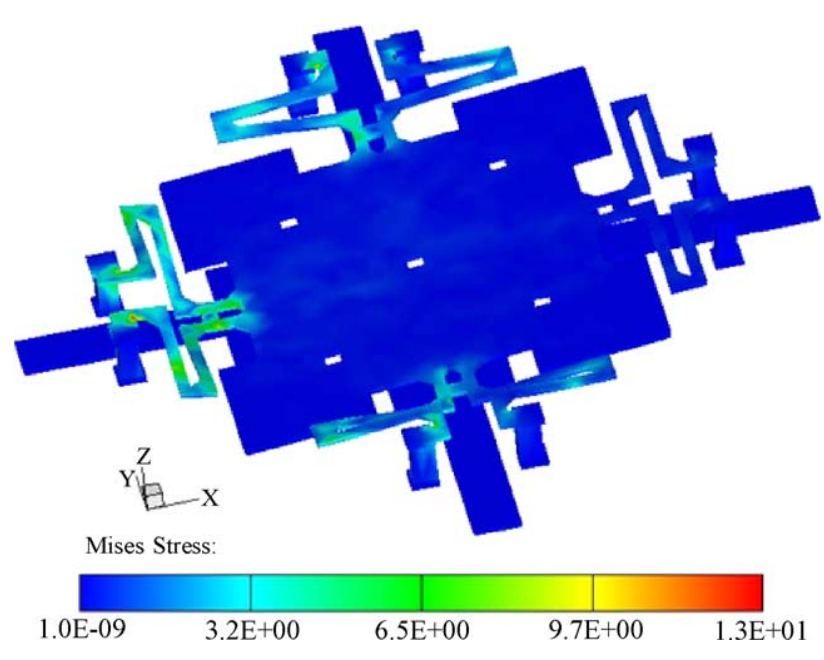

Fig. 3. Stress distribution in a micromirror structure.

$\left(\mathrm{p}^{+}\right.$doped) region in the silicon substrate to yield the same electrical potential between mirror plate and $\mathrm{p}^{+}$doped region.

For the operation of proposed micromirror, in the unactuated state, the mirror plate exhibits no action in relation to the fixed electrode plate, accounting for the fact that the angle between the incident and reflective light beams is almost the same because aluminum is highly optically reflective (>90\%) [15]. No other metal coating add-on process is required in this work, since the mirror plate is made by aluminum and the deposited oxide layer beneath the aluminum is polished by chemical-mechanical-polishing (CMP) in CMOS process. In the actuated state, a voltage is applied to a fixed electrode plate and to the top mirror plate. The mirror plate is pulled down by the electrostatic force, which changes the direction of the reflective light. The micromirror has four operating modes, because of the set-up of four parallel-plate electrode sets.

The electrostatic force, $F_{\mathrm{s}}$, generated between the plates by the applied actuating voltage, $V_{\mathrm{DC}}$, can be derived by con-

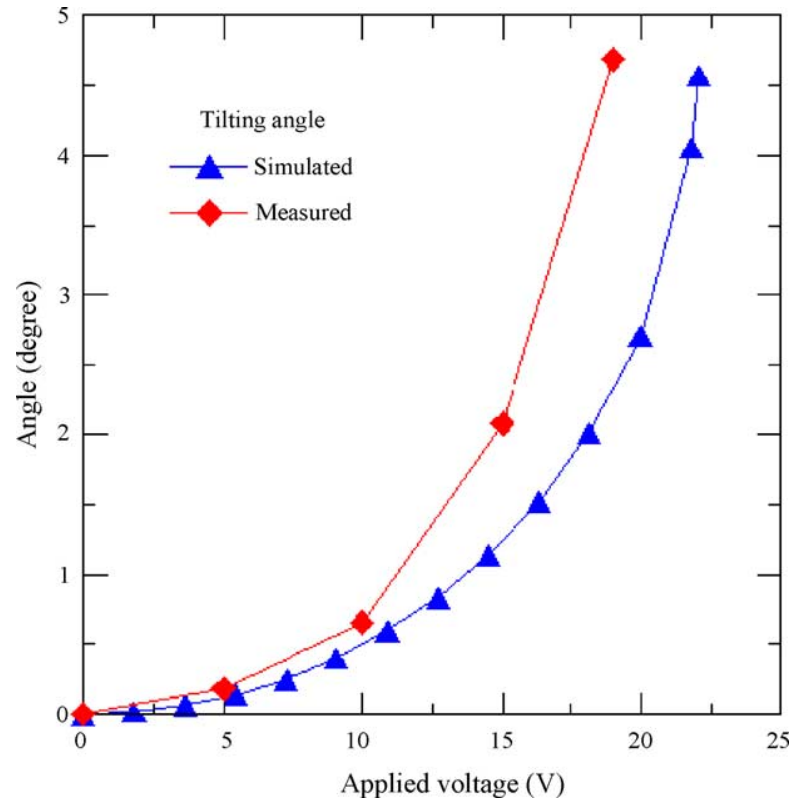

Fig. 4. Angular displacement vs. applied voltage.

sidering the energy, $E$, stored between the plates

$F_{\mathrm{s}}=\frac{\partial E}{\partial d}=\frac{1}{2} \frac{\partial C}{\partial d} V_{\mathrm{DC}}^{2}=\frac{1}{2} \frac{\varepsilon_{\mathrm{r}} \varepsilon_{0} A V_{\mathrm{DC}}^{2}}{d^{2}}$

where $A$ is the effective area of the plates, $d$ the air gap between the two plates, $\varepsilon_{0}$ and $\varepsilon_{\mathrm{r}}$ the permittivity of free space and the relative permittivity, respectively. Hence, a normal out-of-plane force, $F_{\mathrm{s}}$, is applied across an air gap, $d$, between the fixed electrode plate and the top mirror plate, resulting in a deflection.

The finite element method software, CoventorWare, is utilized to simulate the behaviors of the mirror. Fig. 3 illustrates the stress distribution of the micromirror in the actuated state. The deformation in the actuated state almost wholly occurs in the S-shaped flexures of the supported beams. The maximum stress, $13 \mathrm{MPa}$, is below the yield strength of aluminum,

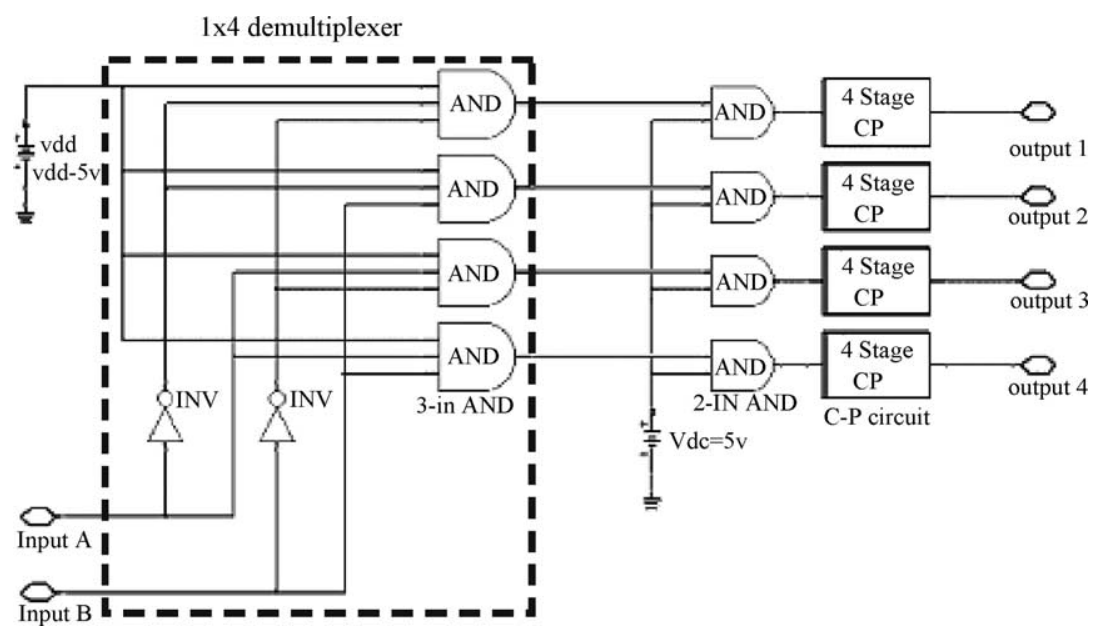

Fig. 5. Schematic design of micromirror control circuit. 


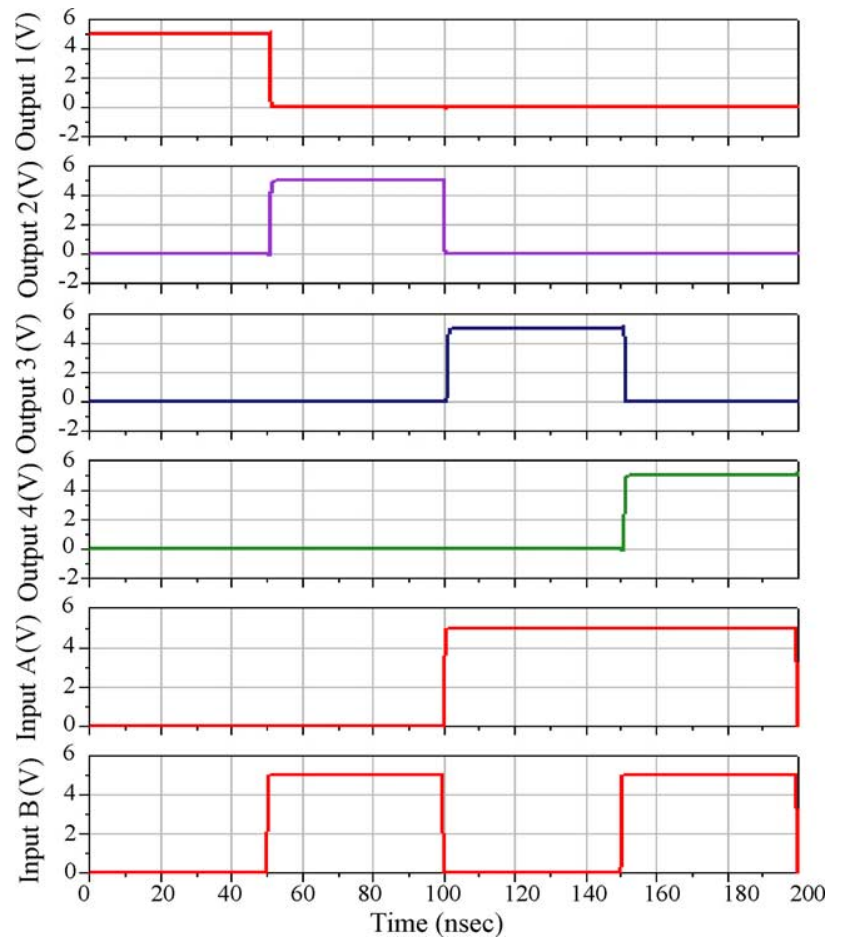

Fig. 6. Simulation of a $1 \times 4$ demultiplexer.

about $90 \mathrm{MPa}$, and thus the deformation of the micromirror can be operated in elastic range. Fig. 4 presents the relationship between the applied voltage and the tilting angle of the mirror plate. The maximum tilting angle of the micromirror is about $5^{\circ}$ at a voltage of $22.5 \mathrm{~V}$. The measured tilting angle deviates from the simulated tilting angle at the applied voltages above $10 \mathrm{~V}$. This deviation is attributed mainly to the slightly softening of the supported beams after ion-bombardment in the reactive ion etching (RIE) processing step or the uncertainly in the exact geometry of the micromirror in the foundry process.

This work uses a simple method to drive the micromirror by a control circuit, as shown in Fig. 5 . The circuit has a $1 \times 4$ demultiplexer digital circuit and four four-stage charge pump circuits. Fig. 6 displays the simulated relationship between the input and output voltage of the $1 \times 4$ demultiplexer. The first four signal curves correspond to the output ports (outputs 1, 2, 3 and 4), which are connected to the four bottom-fixed electrode plates (bottom electrode pads 1, 2, 3 and 4) in the mirror, respectively. The last two signal curves are the input signal. For example, if the input signal is set to $A=1$ (high) and $B=0$ (low), then the output signal will be sent into output port 2. A voltage signal would be then applied to the bottomfixed electrode plate (pad 2) to pull down the mirror plate.

\section{Device fabrication}

The micromachined micromirror is developed according to the Taiwan Semiconductor Manufacture Company

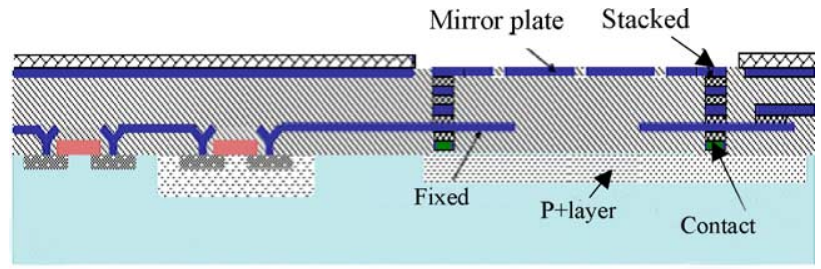

Dry etching

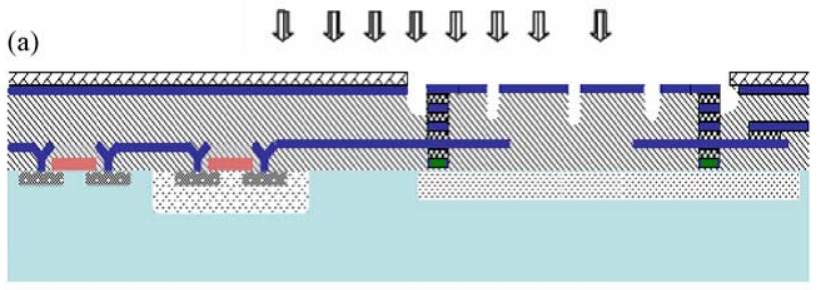

Wet etching

(b)

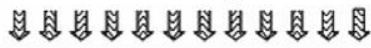

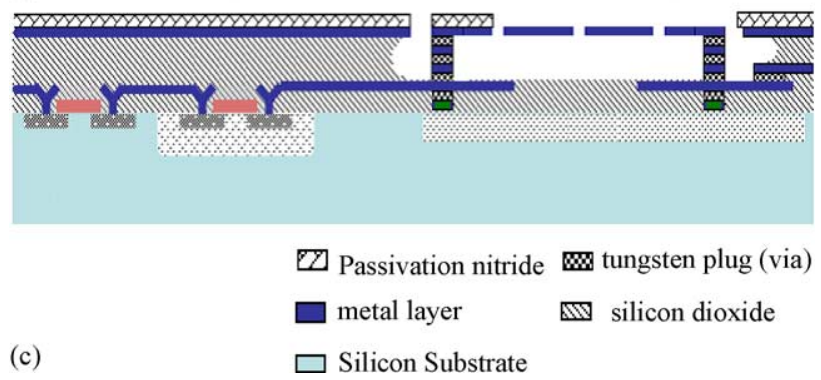

Fig. 7. Schematic process flow: (a) completion of the CMOS foundry process, (b) etching silicon dioxide layer by RIE and (c) removing silicon dioxide layer by BOE/IPA solutions.

(TSMC) CMOS foundry service and design rules. All postprocess procedures require only maskless dry and wet etching and thus avoid the sticking problem. The post-CMOS process has two major steps. The process flow in Fig. 7 illustrates the development of CMOS compatible micromirror structure in cross-section. Fig. 7(a) describes the schematic cross-sections view after TSMC process. Parts of the passivation nitride on the chip were removed in advance, and the top metal layer was exposed. The oxide layers under the mirror plate and the supported beams are sacrificial layers. Two main steps are involved in the post-CMOS process. Fig. 7(b) illustrates the use of anisotropic dry etching with $\mathrm{CF}_{4} / \mathrm{O}_{2}$ to etch the sacrificial oxide layers. This step removes some of the silicon dioxide beneath the top metal layer and forms some cavities through the etching holes. The suspended structures of the micromirror are not all released. Accordingly, isotropic wet etching of oxide must be performed to release the incomplete suspended parts, as shown in Fig. 7(c). This step was completed using BOE (six parts $40 \% \mathrm{NH}_{4} \mathrm{~F}$ and one part 49\% HF) and isopropyl alcohol solution (IPA). Critically, this step enhances the etching selectivity between aluminum and silicon dioxide during wet etching by adding IPA to BOE solution. The suspended structure of the micromirror is released completely, and no sticking occurs after the overall 

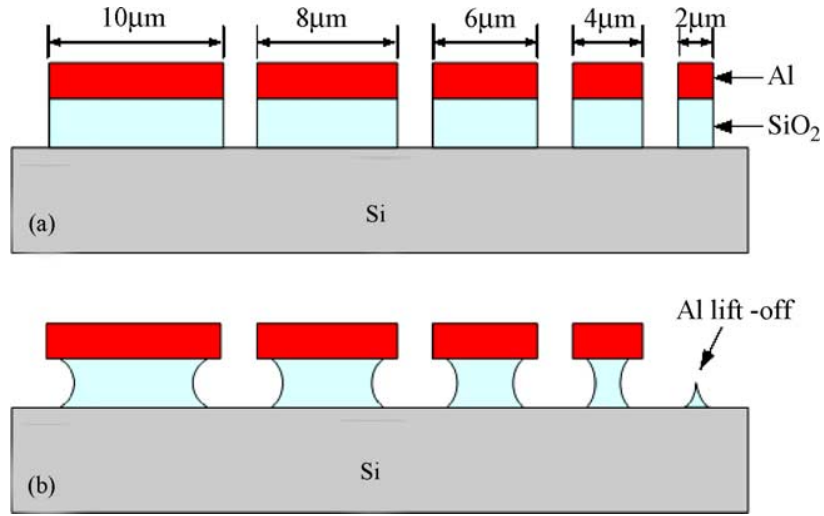

Fig. 8. The lateral etch-rate test-key of BOE for the silicon dioxide: (a) before test and (b) during test.

post-processing. However, the etch time, $40 \mathrm{~min}$, must be precisely controlled to prevent a short circuit which could be caused by leaving a thin oxide layer to cover the bottomelectrode plates.

Fig. 8 depicts a test-key for detecting the lateral etchrate of BOE for silicon dioxide. The test-key as shown in Fig. 8(a) contains five elements with different dimensions: $2 \mu \mathrm{m} \times 2 \mu \mathrm{m}, 4 \mu \mathrm{m} \times 4 \mu \mathrm{m}, 6 \mu \mathrm{m} \times 6 \mu \mathrm{m}, 8 \mu \mathrm{m} \times 8 \mu \mathrm{m}$ and $10 \mu \mathrm{m} \times 10 \mu \mathrm{m}$. Each element is composed of the silicon dioxide and the aluminum layer, and the silicon dioxide layer is under the aluminum layer. Fig. 8(b) illustrates that the silicon dioxide under the aluminum layer generates the lateral etching during the test-key etched by BOE. The lateral etch-rate of BOE for the silicon dioxide can be estimated in accordance with the required time of each aluminum layer to be lift-off in the test-key. For example, the lateral etching distance is $1 \mu \mathrm{m}$ if the aluminum layer (area of $2 \mu \mathrm{m} \times 2 \mu \mathrm{m}$ ) generates lift-off, and recording the etch-time. Fig. 9 reveals the relation between the lateral etch-distance and the etch-time for the silicon dioxide etched by BOE. The lateral etch-rate of BOE for the silicon dioxide evaluated by the experimental results (Fig. 9) was about $8500 \AA / \mathrm{min}$. On the other hand, the etch-rate of $\mathrm{BOE}$ for the aluminum was investigated by measuring the surface profile of the aluminum

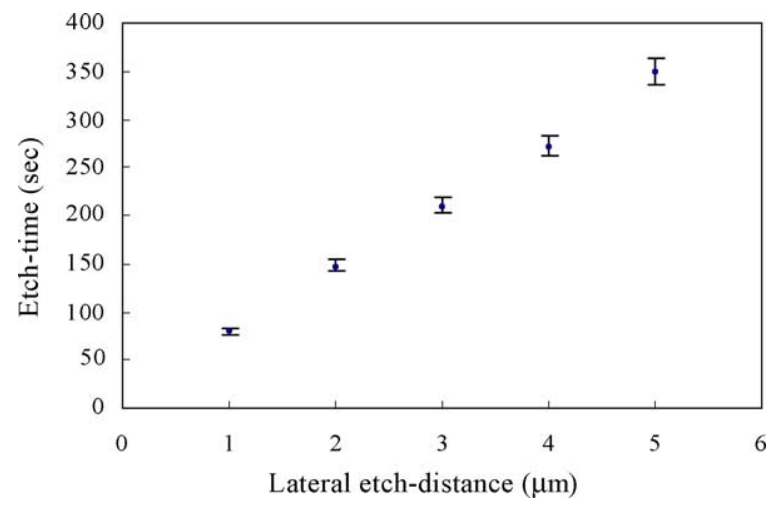

Fig. 9. The lateral etch-distance vs. the etch-time for the silicon dioxide etched by BOE.
Table 1

Experiment parameters of the dry and wet etching steps

Etch recipes for the sacrificial oxide

\begin{tabular}{|c|c|c|}
\hline \multicolumn{2}{|l|}{ Dry etching } & \multirow{2}{*}{$\begin{array}{l}\text { BOE/IPA wet etching } \\
\mathrm{NH}_{4} \mathrm{~F}(40 \%)+\mathrm{HF}(49 \%), 6: 1\end{array}$} \\
\hline $\mathrm{CF}_{4}+\mathrm{O}_{2}$ & & \\
\hline Pressure (mTorr) & 10 & Steps: \\
\hline Power (W) & 180 & $\begin{array}{l}\text { (1) Acetone }+\mathrm{H}_{2} \mathrm{O}, 30 \mathrm{~min} \\
\text { (clean process) }\end{array}$ \\
\hline Gas flow (sccm) & $16.8 \mathrm{CF}_{4}+4 \mathrm{O}_{2}$ & $\begin{array}{l}\text { (2) BOE:IPA (9:1), } 40 \mathrm{~min} \\
\text { (etching step) }\end{array}$ \\
\hline Temperature $\left({ }^{\circ} \mathrm{C}\right)$ & 25 & $\begin{array}{l}\text { (3) } \mathrm{IPA}, 15 \mathrm{~min} \\
\text { (4) } \mathrm{H}_{2} \mathrm{O}, 5 \mathrm{~s} \text { (remove reaction } \\
\text { product on the top metal } \\
\text { surface) } \\
\text { (5) IPA (or acetone), } 15 \mathrm{~min} \\
\text { (rinse) } \\
\text { (6) Hot bake }\left(120^{\circ} \mathrm{C} \text { for }\right. \\
20 \mathrm{~min} \text { ) }\end{array}$ \\
\hline Etch rate $\left(\AA \min ^{-1}\right)$ & $\sim 900$ & $\begin{array}{l}\sim 8500 \text { (low etch rate but } \\
\text { good selectivity) }\end{array}$ \\
\hline
\end{tabular}

layer on the chip. First, the thickness of the aluminum layer measured by the surface profile was $2.20 \mu \mathrm{m}$. Then, the aluminum layer was immersed in BOE with 10, 20 and $30 \mathrm{~min}$, and the thickness of the aluminum layer reduced as 2.18 , 2.15 and $2.08 \mu \mathrm{m}$, respectively. The average etch-rate of BOE for the aluminum evaluated by the measurement was about $315 \AA / \mathrm{min}$. According to the etch-rate of BOE for the silicon dioxide and the aluminum, the etching selectivity could be expressed as 27:1.

Table 1 summarizes the dry and wet etching steps along with the experimental parameters. The ratio of tetrafluoromethane $\left(\mathrm{CF}_{4}\right)$ and oxygen $\left(\mathrm{O}_{2}\right)$ is the decisional recipe when $\mathrm{CF}_{4} / \mathrm{O}_{2}$ RIE are applied to etch silicon dioxide anisotropically. Combining lower $\mathrm{O}_{2}$ flow and low pressure produces a thin polymer passivating film on the silicon dioxide sidewall that inhibits lateral etching. As for the wet etching step in the final procedure, it is well known that one of the most difficult fabrication steps in surface micromachining is the sacrificial etch step and subsequent releasing of the moveable structures. Especially when aluminum is used as the main structures or the interconnect layers it is difficult to selectively etch the sacrificial oxide without attacking the aluminum. Traditional methods to prevent aluminum attack during sacrificial oxide etching are the protection of the metallization using photo-resist (PR) and the use of alternative etch solution like 'pad-etch' [16] or HF vapor etching technique. However, these methods have disadvantages: PR protection is only possible when etch time is limited to several minutes due to adhesion problems. Special etch mixtures or methods can offer reasonable selectivity, but have a low oxide etch rate (pad-etch) and without a standard set-up of the etching process (HF vapor etching technique). In this work, an approach to etch sacrificial oxide with special procedure is proposed, as illustrated in Table 1. Addition of IPA to the BOE is preferred since this makes it possible to maintain a liquid film on the surface of the microstructures when they 


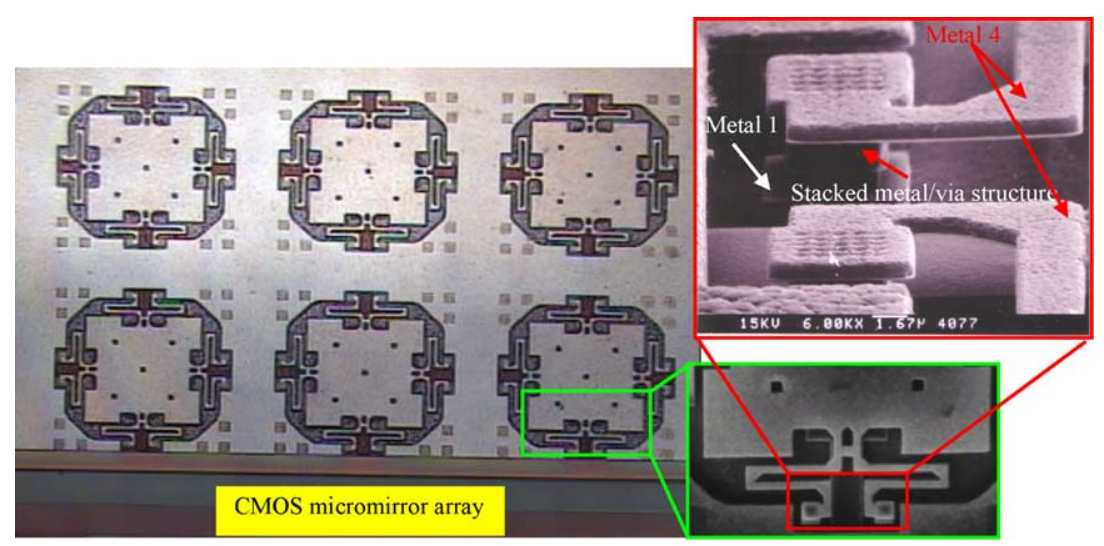

Fig. 10. Pictures of the micromirrors after the post-CMOS process.

are taken out of the etching solution. The CMOS-MEMS chip should not be rinse in water for a long time, because water addition to the HF-based solution will result in rapid attack of the surface of aluminum due to the violent chemical reaction between hydrogen and oxygen ions. Fig. 10 shows some photographs of the micromirror structure following the post-CMOS process by SEM (scanning electron microscope) or optical microscope.
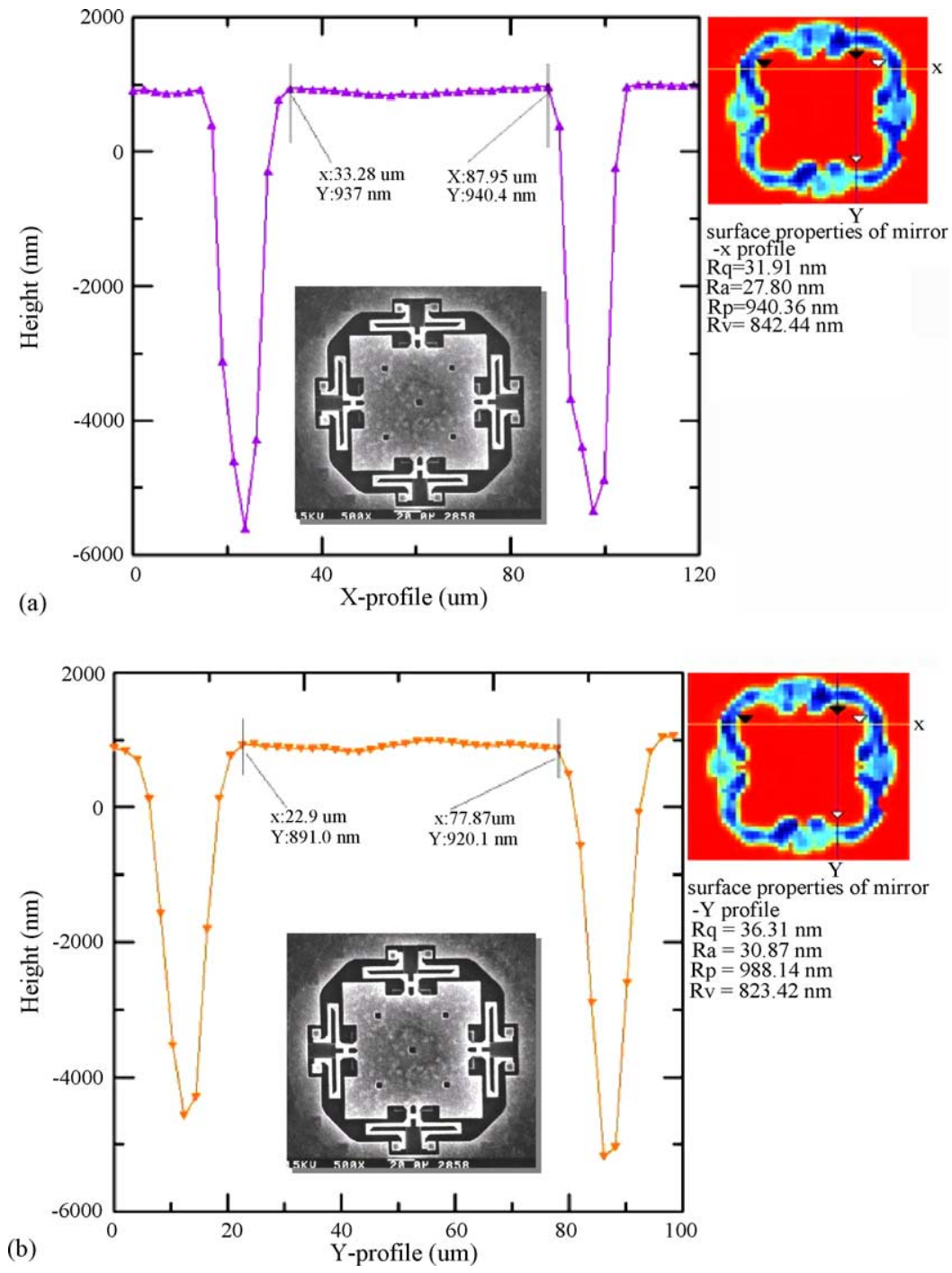

Fig. 11. Surface quality of the mirror plate obtained by WYKO interference measurement system: (a) $X$-direction profile and (b) $Y$-direction profile. 


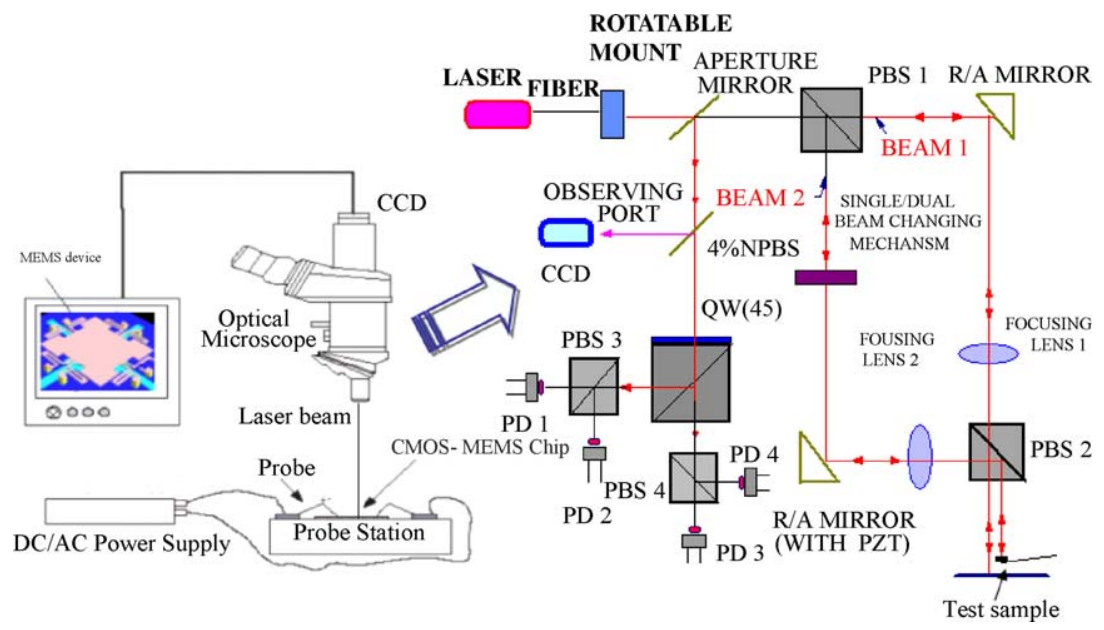

Fig. 12. Schematic optical measurement device (AVID) for the CMOS micromirror device.
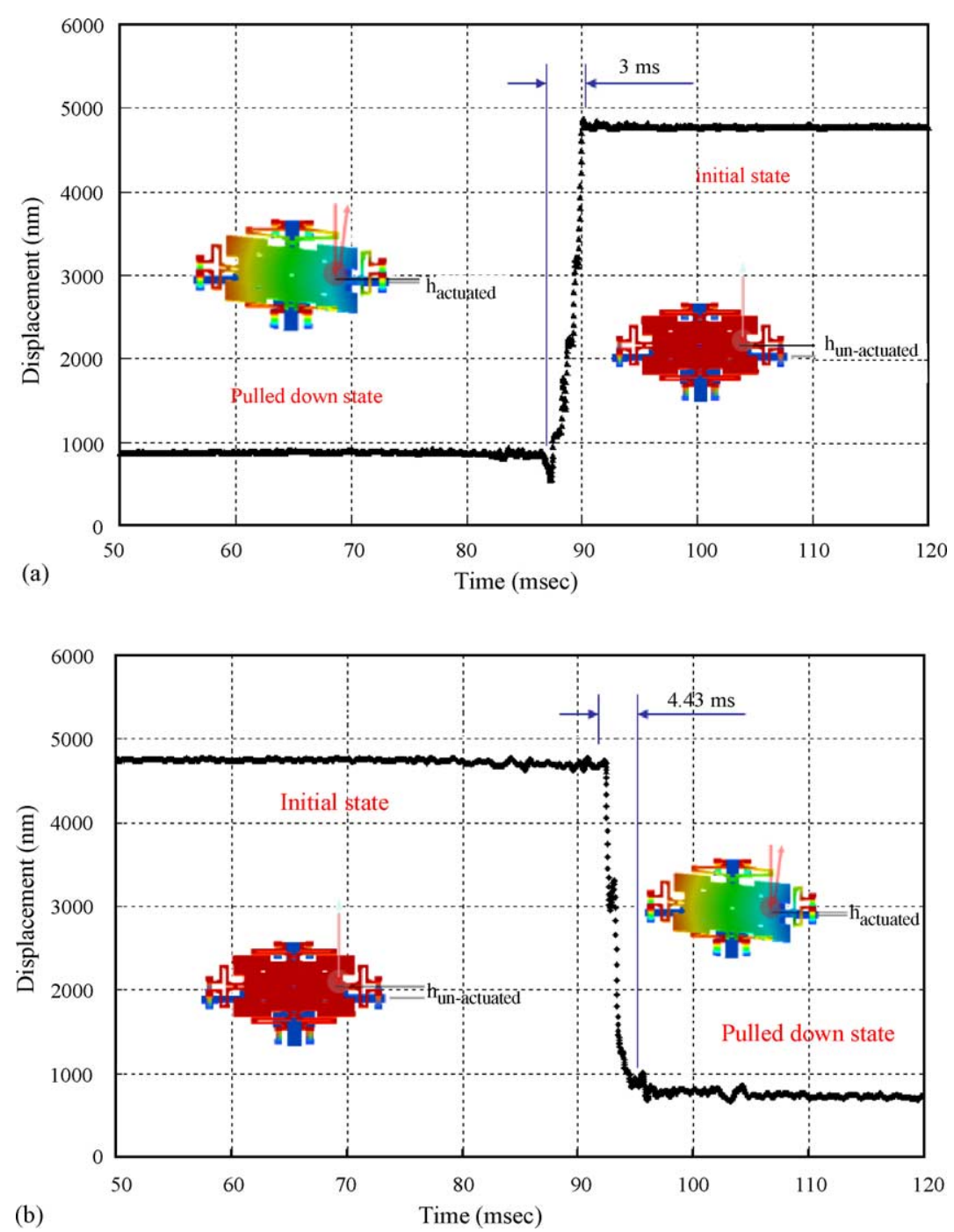

Fig. 13. The switching time of a micromirror: (a) rise time $(3 \mathrm{~ms})$ at the voltage released and (b) fall time ( $4.3 \mathrm{~ms})$ with respect to the operation voltage (22.5 V). 
To reduce the operation voltage and to improve the performance, the S-shaped flexures must be bended or rotated like a pin joint-like mechanism and, in so doing, move smoothly and farther than otherwise. In the CMOS foundry service adopted herein, the via layer constitutes a tunnel in the oxide layer between the two metal layers. The use of the via layer to connect each metal layer not only expands the electrode, but also acts as an anchor, fixing the microstructure onto the silicon substrate. The suspended mirror plate and supported beams are made of the top metal layer (metal 4). Many etching holes are also designed in the mirror plate to reduce the etching time during the process.

\section{Experimental results}

The surface quality of the mirror is also the important determiner of the success of various optical applications that requires a reflective type mirror with a surface roughness of better than one-tenth of the wavelength and a larger radius of curvature, to ensure the flatness of the mirror. In this investigation, the surface roughness and curvature of the mirror were determined using an instrumental interferometer, the WYKO MHT III system, a non-contact, three-dimensional optical interferometric profiler. The root-mean-square (R.M.S.), average roughness $\left(R_{\mathrm{a}}\right)$ and radius of curvature $(\rho)$ of the surface were measured at $31.91,27.80 \mathrm{~nm}$ and $58 \mathrm{~mm}$ in the $X$ direction, and $36.31,30.87 \mathrm{~nm}$ and $49 \mathrm{~mm}$ in the $Y$-direction, respectively, as shown in Fig. 11(a) and (b). These measurements reveal that the surface scattering is acceptable for commonly used reflective-type optical components and verifies the compatibility between the standard IC process and the post-CMOS micromachining technology.

Fig. 12 shows the experimental set-up of the CMOS-MEMS micromirror. The top mirror plate and bottom-fixed electrode plate of the micromirror are connected to the grounded pad and the positive power pad, respectively, of the power supply system. A square-wave with peak voltage of $22.5 \mathrm{~V}$ is applied to the mirror by the circuit. The top mirror plate begins actuation around $15 \mathrm{~V}$, as observed under a microscope. The switching dynamics is measured using a commercial laser Doppler vibrometer (LDV) system (AVID). The response time is shown in Fig. 13(a) and (b). The rise time, corresponding to the recovery state of the micromirror, is about $3 \mathrm{~ms}$ and, the fall time, corresponding to the pulled-down state is $4.3 \mathrm{~ms}$. The response time is fast enough for several MEMS micromirror applications.

\section{Conclusion}

This work presents the design and fabrication of a micromachined micromirror by a fully compatible CMOS process. Experimentally, in this study we perform maskless etching process and obtain good results, including high selectivity in wet etching and full release without damages of the structure or circuit. The lateral etch-rate of BOE for the silicon dioxide was about $8500 \AA / \mathrm{min}$, and the etch-rate of BOE for the aluminum was approximately $315 \AA / \mathrm{min}$. Hence, the etching selectivity of $\mathrm{BOE}$ for the silicon dioxide and the aluminum was $27: 1$.

The micromirror has a tilting angle of approximately $5^{\circ}$ at an operation voltage around $22.5 \mathrm{~V}$. The switching times are 3 and $4.3 \mathrm{~ms}$ for the rise and the fall times, respectively. The whole procedure is simple and compatible with the CMOS process. This investigation not only reduces the developing time, but also minimizes the scale by following the advancing CMOS process. The micromirror proposed herein has a monolithic integration capability with circuits and is potentially for future applications in optics or other fields by the advanced VLSI process. For instance, some VLSI foundries have provided the thick top metal layer ( 2 or $3 \mu \mathrm{m}$ ) already for mixed signal or RF circuit. That will be a good option to develop CMOS-MEMS devices with better material property and device performance.

\section{Acknowledgements}

The authors would like to thank Song-Nan Chen, Yao-Tian Huang, Wei-Feng Chien of the Institute of Applied Mechanics, National Taiwan University, Professor Chien-Liu Chang for their valuable advice and assistance in experiment. In addition, we would, finally, like to thank the NSC Northern Region MEMS Research Center, the National Chip Implementation Center and the National Center for High-performance Computing for kindly making their complete research facilities available.

\section{References}

[1] M. Parameswaran, A.M. Robinson, D.L. Blackburn, M. Gaitan, J. Geist, Micromachined thermal radiation emitter from a commercial CMOS process, IEEE Electron Device Lett. 12 (1991) 57-59.

[2] V. Milanovic, M. Ozgur, D.C. DeGroot, J.A. Jargon, M. Gaitan, M.E. Zaghloul, Characterization of broad-band transmission for coplanar waveguides on CMOS silicon substrates, IEEE Trans. Microw. Theory Tech. 46 (1998) 632-640.

[3] L.J. Hornbeck, Digital light processing and MEMS: an overview advanced applications of lasers in materials processing, in: Summer Topical Meetings on Broadband Optical Networks/Smart Pixels/Optical MEMS and their Applications, 1996, pp. 7-8.

[4] D.W. Monk, Digital light processing: a new image technology for the television of the future, Int. Broadcasting Convention (1997) 581-586.

[5] D. Hah, C.A. Choi, C.H. Jun, Y.T. Kim, A self-aligned vertical combdrive actuator using surface micromachining for scanning micromirrors, in: Proceedings of the IEEE/LEOS International Conference on Optical MEMS, 2003, pp. 151-152.

[6] N. Konno, M. Tsugai, E. Yoshikawa, T. Usami, R. Fujita, Design optimization and improved performance on the link beam driven, miniaturized scanning mirror device, in: Proceedings of the IEEE/LEOS International Conference on Optical MEMS, 2000, pp. 11-12. 
[7] C.H. Kim, N. Park, Y.K. Kim, Reflective-type variable optical attenuator using off-axis misalignment, in: Proceedings of the IEEE/LEOS International Conference on Optical MEMS, 2002, pp. 55-56.

[8] M.S. Yang, Y.O. Noh, Y.H. Won, W.Y. Hwang, Very low crosstalk $1 \times 2$ digital optical switch integrated with variable optical attenuators, Electron. Lett. 37 (2001) 587-588.

[9] C.R. Giles, V. Aksyuk, B. Barber, R. Ruel, L. Stulz, D. Bishop, A silicon MEMS optical switch attenuator and its use in lightwave subsystems, IEEE J. Quant. Electron. 5 (1999) 18-25.

[10] K.N. Lee, D.S. Shin, Y.S. Lee, Y.K. Kim, Micromirror array for protein microarray fabrication, J. Micromech. Microeng. 13 (2003) 474-481.

[11] L.Y. Lin, E.L. Goldstein, R.W. Tkach, Angular-precision enhancement in free-space micromachined optical switches, IEEE Photonics Tech. Lett. 11 (1999) 1253-1255.

[12] H. Toshiyoshi, H. Fujita, Electrostatic microtorsion mirrors for an optical switch matrix, J. Microelectromech. Syst. 5 (1996) 231-237.

[13] L. Houlet, P. Helin, T. Bourouina, G. Reyne, E. Diffour-Gergam, H. Fujita, Movable vertical mirror arrays for optical microswitch matrixes and their electromagnetic actuation, IEEE J. Quant. Electron. 8 (2002) 58-63.

[14] H. Toshiyoshi, H. Fujita, An electrostatically operated torsion mirror for optical switching device, in: Proceedings of the Eighth International Conference on Solid-State Sensors and Actuators, 1995, pp 297-300.

[15] C. Marxer, C. Thio, M.A. Gretillat, N.F. de Rooij, R. Battig, O. Anthamatten, B. Valk, P. Vogel, Vertical mirrors fabricated by deep reactive ion etching for fiber-optic switching applications, J. Microelectromech. Syst. 6 (1997) 277-285.

[16] J. Buhler, J. Funk, P. Steiner, P.M. Sarro, H. Baltes, Double pass metallization for CMOS aluminum actuators, Transducers 95 (1996) 360-363.

\section{Biographies}

Ying-Chou Cheng received his MS degree from National Cheng-Kung University, Taiwan in 1999 and $\mathrm{PhD}$ degree from the Department of
Mechanical Engineering of National Taiwan University, Taiwan in 2004. $\mathrm{He}$ is currently a research assistant at the Institute of Applied Mechanics, National Taiwan University with the research interests on IC compatible MEMS and microsensors technologies.

Ching-Liang Dai received the MS degree in the Applied Mechanics from National Taiwan University, Taiwan in 1993, and the PhD degree in the Mechanical Engineering from National Taiwan University in 1997. He is currently an assistant professor at the Department of Mechanical Engineering, National Chung Hsing University, Taiwan. His research interests are CMOS-MEMS, integrated microsensors and mechanical properties of thin films.

Chi-Yuan Lee received his MS degree from Tamkang University, Taiwan in 1997 and $\mathrm{PhD}$ degree from the Department of Mechanical Engineering of National Taiwan University, Taiwan in 2004. He is currently an assistant professor at the Department of Mechanical Engineering, Yuan Ze University, Taiwan. His research interests are MEMS, acoustic device and microfuel cell.

Ping-Hei Chen was born in Taichung, Taiwan on Augest 31, 1958. He received the BSc degree in Mechanical Engineering from National Taiwan University in 1980. He then received the MS and PhD degrees from the University of Minnesota in 1984 and 1988, respectively. Since 1988, he had been an Associate Professor in the Department of Mechanical Engineering at National Taiwan University. He was promoted to the full Professor at 1996, then served as the vice-chairman for 2 years, and chairman for 3 years at Department of Mechanical Engineering in National Taiwan University. His current research interests are MEMS, biochips, diamond film wafer, nanotechnology, thermal and fluid control in MEMS, and packaging for optical devices and IC.

Pei-Zen Chang was born in Chia-Yi, Taiwan, ROC in 1962. He received the BS degree in Civil Engineering from National Taiwan University, Taipei, Taiwan in 1984, and the PhD degree in Theoretical and Applied Mechanics from Cornell University, Ithaca, NY in 1991. His PhD dissertation was about the Mechanics of Superconducting Magnetic Bearings. He joined the faculty of Institute of Applied Mechanics, National Taiwan University in 1991 and became a professor in 1999. His current research interests are in the area of micromachined sensors and actuators. 